\title{
On the subseismic approximation for low-frequency gravity modes in spherically symmetric stars
}

\author{
P. Smeyers ${ }^{\star}$ \\ Instituut voor Sterrenkunde, Katholieke Universiteit Leuven, Celestijnenlaan 200 B, 3001 Heverlee, Belgium
}

Received 6 March 2001 / Accepted 6 April 2001

\begin{abstract}
In a comparative study of the anelastic and the subseismic approximation for the description of lowfrequency gravity modes in spherically symmetric stars, Dintrans \& Rieutord (2001) stress that the subseismic approximation is inconsistent in that the Eulerian perturbation of the mass density is negligible as is the Eulerian perturbation of the pressure and that consequently the equation of mass conservation is not satisfied. In this paper, attention is drawn to the asymptotic theory for low-degree, low-frequency gravity modes of spherically symmetric stars developed by Smeyers et al. (1995). A main conclusion is that, in the main body of a star, the subseismic approximation results as a natural consequence of the lowest-order asymptotic approximation. At the same order of approximation, the Eulerian perturbation of the mass density is derived from the equation of mass conservation and is different from zero. It also follows from the lowest-order asymptotic approximation that the Eulerian perturbation of the pressure is different from zero in a small region near the star's surface, so that the subseismic approximation is not adequate in that region.
\end{abstract}

Key words. stars: oscillations

\section{Introduction}

In a forthcoming paper, Dintrans \& Rieutord (2001) present a comparative study of the anelastic and the subseismic approximation for the description of low-frequency gravity modes in spherically symmetric stars.

The anelastic approximation, in which the Eulerian perturbation of the mass density is set equal to zero, has been extensively used in studies of compressible convection in stars (for references, see Dintrans \& Rieutord). The subseismic approximation rests on the assumption that, for low-frequency gravity modes, the Lagrangian pressure perturbation of a moving mass element is almost entirely determined by the effect of the transport along the equilibrium pressure gradient, so that the Eulerian pressure perturbation may be set equal to zero. It stems from terrestrial seismology (Smylie \& Rochester 1981; Smylie et al. 1984) and was adopted in astrophysics by De Boeck et al. (1992) for an asymptotic treatment of low-frequency gravity modes in spherically symmetric stars. As noted by the latter authors, a similar approximation is commonly adopted in the theory of stellar structure, both in the derivation of the local criterion for stability against convection and in the mixing length theory of convection.

* e-mail: Paul.Smeyers@ster.kuleuven.ac.be
The anelastic and the subseismic approximation have the common property of filtering out the acoustic modes of a spherically symmetric star.

In their study, Dintrans \& Rieutord stress that the subseismic approximation is inconsistent, in that the Eulerian perturbation of the mass density is negligible as is the Eulerian perturbation of the pressure and that consequently the equation of the mass conservation is not satisfied.

Our aim is to draw attention to the asymptotic theory for low-degree, low-frequency gravity modes of spherically symmetric stars developed by Smeyers et al. (1995). A main conclusion of this theory is that, in the main body of a star, the subseismic approximation is a natural consequence of the lowest-order asymptotic approximation. At the same order of approximation, the Eulerian perturbation of the mass density is derived from the equation of mass conservation and is different from zero. It also follows from the lowest-order asymptotic approximation that the Eulerian perturbation of the pressure is different from zero in a small region near the star's surface, so that the subseismic approximation is not adequate in that region.

In order to clarify the matter, we give an outline of the lowest-order asymptotic theory. The asymptotic theory is based on the use of a two-variable expansion procedure in the domain situated at sufficiently large distances from 
the boundary points at $r=0$ and $r=R$, and of boundarylayer techniques in the regions near the boundary points.

The plan of the paper is as follows. In Sect. 2, the basic equations are introduced. In Sect. 3, we present the first-order asymptotic solutions that are uniformly valid from the boundary point at $r=0$ to a sufficiently large distance from the boundary point at $r=R$. In Sect. 4, we similarly present the first-order asymptotic solutions that are uniformly valid from the boundary point at $r=R$ to a sufficiently large distance from the boundary point at $r=0$. In Sect. 5, the equation for the eigenfrequencies is given. Section 6 is devoted to concluding remarks.

\section{Basic equations}

After separation of the time by a factor $\exp (\mathrm{i} \sigma t)$ and of the angular variables $\theta$ and $\phi$ by means of spherical harmonics $Y_{\ell}^{m}(\theta, \phi)$, the equations governing linear, isentropic oscillations of a spherically symmetric star that are associated with an angular frequency $\sigma$ different from zero can be transformed into a system of two differential equations for the radial parts of the divergence and the radial component of the Lagrangian displacement (Pekeris 1938; see also Tassoul 1990). Let these radial functions be $\alpha(r)$ and $\xi(r)$, respectively. The system of differential equations then takes the form

$$
\begin{aligned}
& \frac{\mathrm{d}^{2} \alpha}{\mathrm{d} r^{2}}+K_{2}(r) \frac{\mathrm{d} \alpha}{\mathrm{d} r}+\left[\frac{\sigma^{2}}{c^{2}}+K_{3}(r)+\frac{K_{1}(r)}{\sigma^{2}}\right] \alpha \\
& =-K_{4}(r) \frac{\mathrm{d} \xi}{\mathrm{d} r} \\
& \frac{\mathrm{d}^{2} \xi}{\mathrm{d} r^{2}}+\frac{4}{r} \frac{\mathrm{d} \xi}{\mathrm{d} r}-\frac{\ell(\ell+1)-2}{r^{2}} \xi \\
& \quad=\frac{\mathrm{d} \alpha}{\mathrm{d} r}-\left[\frac{c^{2}}{g} \frac{K_{1}(r)}{\sigma^{2}}-\frac{2}{r}\right] \alpha,
\end{aligned}
$$

where

$$
\begin{aligned}
K_{1}(r) & =\ell(\ell+1) \frac{N^{2}}{r^{2}}, \\
K_{2}(r) & =\frac{2}{r}+\frac{2}{\rho c^{2}} \frac{\mathrm{d}\left(\rho c^{2}\right)}{\mathrm{d} r}-\frac{1}{\rho} \frac{\mathrm{d} \rho}{\mathrm{d} r}, \\
K_{3}(r) & =-\frac{\ell(\ell+1)}{r^{2}}+\frac{2 g}{c^{2}}\left(\frac{1}{g} \frac{\mathrm{d} g}{\mathrm{~d} r}+\frac{1}{r}\right) \\
+\frac{1}{\rho c^{2}} & \frac{\mathrm{d}\left(\rho c^{2}\right)}{\mathrm{d} r}\left(\frac{2}{r}-\frac{1}{\rho} \frac{\mathrm{d} \rho}{\mathrm{d} r}\right)+\frac{1}{\rho c^{2}} \frac{\mathrm{d}^{2}\left(\rho c^{2}\right)}{\mathrm{d} r^{2}}, \\
K_{4}(r) & =-\frac{2 g}{c^{2}}\left(\frac{1}{g} \frac{\mathrm{d} g}{\mathrm{~d} r}-\frac{1}{r}\right) .
\end{aligned}
$$

The variables have their usual meaning. For the sake of simplification, $N^{2}$ is assumed to be positive everywhere in the star.

As the small expansion parameter in the asymptotic theory, $\varepsilon=|\sigma|$ is adopted.

\section{Uniformly valid first-order asymptotic solutions from the boundary point at $r=0$}

For the construction of asymptotic solutions in the domain of the star situated at sufficiently large distances from the boundary points at $r=0$ and $r=R$, the fast independent variable

$\tau(r)=\frac{[\ell(\ell+1)]^{1 / 2}}{\varepsilon} \int_{0}^{r} \frac{N\left(r^{\prime}\right)}{r^{\prime}} \mathrm{d} r^{\prime}$

is introduced besides the radial distance $r$, which is considered as a slow independent variable. The boundary-layer coordinate used in the region near $r=0$ corresponds to the fast independent variable $\tau(r)$.

In terms of these variables, the first-order asymptotic solution for the divergence of the Lagrangian displacement that is uniformly valid from $r=0$ to a sufficiently large distance from $r=R$ is given by

$\alpha^{(c)}(r ; \varepsilon)=A_{0, c} \frac{g}{\left(N^{2} r^{6} c^{8} \rho^{2}\right)^{1 / 4}} \tau^{1 / 2} J_{\ell+1 / 2}(\tau)$.

Here $J_{\ell+1 / 2}(\tau)$ is the Bessel function of the first kind of degree $\ell+1 / 2$, and $A_{0, c}$ an undetermined constant.

The first-order asymptotic solution for the radial component of the Lagrangian displacement that is uniformly valid in the same region is given by

$\xi^{(c)}(r ; \varepsilon)=A_{0, c} \frac{1}{\left(N^{2} r^{6} \rho^{2}\right)^{1 / 4}} \tau^{1 / 2} J_{\ell+1 / 2}(\tau)$.

Hence, the relation holds

$\xi^{(c)}(r ; \varepsilon)=\frac{c^{2}}{g} \alpha^{(c)}(r ; \varepsilon)$.

By use of the energy equation written in the form

$P^{\prime}=-\rho c^{2} \alpha-\frac{\mathrm{d} P}{\mathrm{~d} r} \xi$,

and of the condition of hydrostatic equilibrium

$\frac{\mathrm{d} P}{\mathrm{~d} r}=-\rho g$,

it results that

$P^{\prime(c)}(r ; \varepsilon)=0$,

i.e., the first-order asymptotic solution for the Eulerian perturbation of the pressure that is uniformly valid from $r=0$ to a sufficiently large distance from $r=R$ is identically zero.

The uniformly valid first-order asymptotic solution for the Eulerian perturbation of the mass density is derived by means of the equation for the mass conservation

$\rho^{\prime}=-\rho \alpha-\frac{\mathrm{d} \rho}{\mathrm{d} r} \xi$.

It results that

$\rho^{\prime(c)}(r ; \varepsilon)=\rho \frac{N^{2}}{g} \xi^{(c)}(r ; \varepsilon)$. 
The solution reveals that, for a mass element which moves slowly and isentropically in the stellar interior and which adjusts its pressure to the pressure of its surroundings, the relative difference in mass density with the surroundings is determined by the difference between the effect of the transport along the isentropic density gradient and the effect of the transport along the actual density gradient.

The uniformly valid first-order asymptotic solution for the radial part $\eta(r)$ of the transverse component of the Lagrangian displacement is derived by means of the equality

$\alpha=\frac{1}{r^{2}} \frac{\mathrm{d}}{\mathrm{d} r}\left(r^{2} \xi\right)-\frac{\ell(\ell+1)}{r^{2}} \eta$.

By using uniformly valid first-order asymptotic solutions (8) and (9) and restricting the first derivative $\mathrm{d}\left(r^{2} \xi^{(c)}(r ; \varepsilon)\right) / \mathrm{d} r$ to its dominant part, one has

$$
\begin{aligned}
\eta^{(c)}(r ; \varepsilon)= & \frac{A_{0, c}}{\varepsilon} \frac{1}{[\ell(\ell+1)]^{1 / 2}} \\
& \times \frac{N r}{\left(N^{2} r^{6} \rho^{2}\right)^{1 / 4}} \frac{\mathrm{d}}{\mathrm{d} \tau}\left[\tau^{1 / 2} J_{\ell+1 / 2}(\tau)\right] .
\end{aligned}
$$

Hence, at the first-order asymptotic approximation, the ratio of the transverse component to the radial component of the Lagrangian displacement is of the order of $\varepsilon^{-1}$. In the boundary layer near $r=0, \eta^{(c)}(r ; \varepsilon) \propto \varepsilon^{-1} \tau^{\ell}$, and $\xi^{(c)}(r ; \varepsilon) \propto \varepsilon^{-2} \tau^{\ell-1}$, so that the ratio is of the order of $\varepsilon$ and tends to zero as $\tau(r) \rightarrow 0$.

The Eulerian perturbation of the gravitational potential is related to the radial part of the transverse component of the Lagrangian displacement and the Eulerian perturbation of the pressure as

$\Phi^{\prime}=\varepsilon^{2} \eta-\frac{P^{\prime}}{\rho}$.

Consequently, in the region extending from $r=0$ to a sufficiently large distance from $r=R$, the uniformly valid first-order asymptotic solution for the Eulerian perturbation of the gravitational potential, $\Phi^{\prime(c)}(r ; \varepsilon)$, is of the order of $\varepsilon$.

\section{Uniformly valid first-order asymptotic solutions from $r=R$ to a sufficiently large distance from $r=0$}

Near the boundary point at $r=R$, boundary-layer solutions are constructed with the supposition that the mass density may be expanded in a Taylor series as

$\rho=\rho_{\mathrm{s}} z^{n_{\mathrm{e}}}[1+O(z)]$

where $z=1-r / R$, and $\rho_{\mathrm{s}}$ is a constant different from zero. The exponent $n_{\mathrm{e}}$ corresponds to the effective polytropic index of the superficial layers. Moreover, the boundarylayer coordinate

$$
\begin{aligned}
\tau_{\mathrm{s}}(r) & =\frac{[\ell(\ell+1)]^{1 / 2}}{\varepsilon} \int_{r}^{R} \frac{N\left(r^{\prime}\right)}{r^{\prime}} \mathrm{d} r^{\prime} \\
& =\frac{[\ell(\ell+1)]^{1 / 2} R}{\varepsilon} \int_{0}^{z} \frac{N\left(r^{\prime}\right)}{r^{\prime}} \mathrm{d} z^{\prime}
\end{aligned}
$$

is introduced, which obeys the relation

$\tau_{\mathrm{s}}(r)=\tau_{R}-\tau(r)$

with

$\tau_{R}=\frac{[\ell(\ell+1)]^{1 / 2}}{\varepsilon} \int_{0}^{R} \frac{N\left(r^{\prime}\right)}{r^{\prime}} \mathrm{d} r^{\prime}$.

The first-order asymptotic solution for the divergence of the Lagrangian displacement that is uniformly valid from $r=R$ to a sufficiently large distance from $r=0$ is expressed in terms of the coordinate $\tau_{\mathbf{s}}(r)$ and the radial coordinate $r$ as

$\alpha^{(s)}(r ; \varepsilon)=A_{0, \mathrm{~s}} \frac{g}{\left(N^{2} r^{6} c^{8} \rho^{2}\right)^{1 / 4}} \tau_{\mathrm{s}}^{1 / 2} J_{n_{\mathrm{e}}+1}\left(\tau_{\mathrm{s}}\right)$.

Here $J_{n_{\mathrm{e}}+1}\left(\tau_{\mathrm{s}}\right)$ is the Bessel function of the first kind of degree $n_{\mathrm{e}}+1$, and $A_{0, \mathrm{~s}}$ an undetermined constant. As $\tau_{s} \rightarrow 0$,

$\alpha^{(\mathrm{s})}(r ; \varepsilon) \propto \varepsilon^{-\left(n_{\mathrm{e}}+3 / 2\right)} \tau_{\mathrm{s}}^{0}$,

so that the divergence of the Lagrangian displacement remains finite.

The first-order asymptotic solution for the radial component of the Lagrangian displacement that is uniformly valid in the same region is given by

$\xi^{(\mathrm{s})}(r ; \varepsilon)=A_{0, \mathrm{~s}} \frac{1}{\left(N^{2} r^{6} \rho^{2}\right)^{1 / 4}} \tau_{\mathrm{s}}^{1 / 2} J_{n_{\mathrm{e}}+1}\left(\tau_{\mathrm{s}}\right)$
$\quad \times\left\{1-\frac{2 \mathcal{N}_{\mathrm{s}}^{2}}{\tau_{\mathrm{s}}^{2}}\left[\frac{\mathrm{d} \ln J_{n_{\mathrm{e}}+1}\left(\tau_{\mathrm{s}}\right)}{\mathrm{d} \ln \tau_{\mathrm{s}}}+\left(n_{\mathrm{e}}+1\right)\right]\right\}$.

Here $\mathcal{N}_{\mathrm{s}}^{2}$ is a constant defined as

$\mathcal{N}_{\mathrm{s}}^{2}=n_{\mathrm{e}}-\frac{n_{\mathrm{e}}+1}{\Gamma_{1, R}}$,

where $\Gamma_{1, R}$ is the value of the generalized isentropic coefficient $\Gamma_{1}$ at $r=R$. As $\tau_{\mathrm{s}}(r) \rightarrow 0$,

$\xi^{(s)}(r ; \varepsilon) \propto \varepsilon^{-\left(n_{e}-1 / 2\right)} \tau_{\mathrm{s}}^{0}$,

so that the radial component of the Lagrangian displacement too remains finite.

First-order asymptotic solutions (25) and (23) are related as

$\xi^{(s)}(r ; \varepsilon)=\frac{c^{2}}{g} \alpha^{(s)}(r ; \varepsilon)$

$\times\left\{1-\frac{2 \mathcal{N}_{\mathrm{s}}^{2}}{\tau_{\mathrm{s}}^{2}}\left[\frac{\mathrm{d} \ln J_{n_{\mathrm{e}}+1}\left(\tau_{\mathrm{s}}\right)}{\mathrm{d} \ln \tau_{\mathrm{s}}}+\left(n_{\mathrm{e}}+1\right)\right]\right\}$. 
The uniformly valid first-order asymptotic solution for the Eulerian perturbation of the pressure is then given by

$$
\begin{aligned}
P^{\prime(\mathrm{s})}(r ; \varepsilon)= & -\rho c^{2} \frac{2 \mathcal{N}_{\mathrm{s}}^{2}}{\tau_{\mathrm{s}}^{2}} \\
& \times\left[\frac{\mathrm{d} \ln J_{n_{\mathrm{e}}+1}\left(\tau_{\mathrm{s}}\right)}{\mathrm{d} \ln \tau_{\mathrm{s}}}+\left(n_{\mathrm{e}}+1\right)\right] \alpha^{(\mathrm{s})}(r ; \varepsilon) .
\end{aligned}
$$

As $\tau_{\mathrm{s}}(r) \rightarrow 0$,

$\frac{P^{\prime(\mathrm{s})}(r)}{\rho} \propto \varepsilon^{-\left(n_{\mathrm{e}}-1 / 2\right)} \tau_{\mathrm{s}}^{0}$

Apart from the particular case in which $n_{\mathrm{e}}=$ $\left(F_{1, R}-1\right)^{-1}$, the Eulerian perturbation of the pressure is thus different from zero near the star's surface, so that the asymptotic approximation does not yield the subseismic approximation in that region.

For larger values of $\tau_{\mathrm{s}}(r)$, the Bessel function $J_{n_{\mathrm{e}}+1}\left(\tau_{\mathrm{s}}\right)$ and its first derivative may be approximated by their asymptotic forms. It then follows that

$\lim _{\tau_{s} \rightarrow \infty} \frac{1}{\tau_{\mathrm{s}}^{2}}\left[\frac{\mathrm{d} \ln J_{n_{\mathrm{e}}+1}\left(\tau_{\mathrm{s}}\right)}{\mathrm{d} \ln \tau_{\mathrm{s}}}+\left(n_{\mathrm{e}}+1\right)\right]=0$.

Hence, the uniformly valid first-order asymptotic solution $P^{\prime(\mathrm{s})}(r ; \varepsilon)$ tends to zero for larger values of $\tau_{\mathrm{s}}(r)$, in accordance with the uniformly valid first-order asymptotic solution derived for the region from $r=0$ to a sufficiently large distance from $r=R$.

By deriving the uniformly valid first-order asymptotic solution for the Eulerian perturbation of the mass density by means of Eq. (14) for the mass conservation, one has

$$
\begin{aligned}
& \rho^{\prime(\mathrm{s})}(r ; \varepsilon)=-\alpha^{(\mathrm{s})}(r ; \varepsilon)\left\{\rho+\frac{\mathrm{d} \rho}{\mathrm{d} r} \frac{c^{2}}{g}\right. \\
& \left.\quad \times\left\{1-\frac{2 \mathcal{N}_{\mathrm{s}}^{2}}{\tau_{\mathrm{s}}^{2}}\left[\frac{\mathrm{d} \ln J_{n_{\mathrm{e}}+1}\left(\tau_{\mathrm{s}}\right)}{\mathrm{d} \ln \tau_{\mathrm{s}}}+\left(n_{\mathrm{e}}+1\right)\right]\right\}\right\} .
\end{aligned}
$$

The uniformly valid first-order asymptotic solution for the radial part of the transverse component of the Lagrangian displacement is derived by means of Eq. (16). As $\tau_{\mathrm{s}}(r) \rightarrow 0$,

$\eta^{(\mathrm{s})}(r ; \varepsilon) \propto \varepsilon^{-\left(n_{\mathrm{e}}+3 / 2\right)} \tau_{\mathrm{s}}^{0}$.

Hence, $\eta^{(\mathrm{s})}(r ; \varepsilon)$ is of the order of $\varepsilon^{-1}$ and becomes of the order of $\varepsilon^{-\left(n_{\mathrm{e}}+3 / 2\right)}$ as $\tau_{\mathrm{s}} \rightarrow 0$. Correspondingly, the ratio of the transverse component to the radial component of the Lagrangian displacement is of the order of $\varepsilon^{-1}$ and becomes of the order of $\varepsilon^{-2}$ as $\tau_{\mathrm{s}}(r) \rightarrow 0$. It may be noticed that, from the centre to the surface of the star, the ratio increases by three orders of magnitude in the small expansion parameter $\varepsilon$.

Finally, the first-order asymptotic solution for the Eulerian perturbation of the gravitational potential is again derived by means of Eq. (18). In the boundary layer near $r=R$, the two dominant terms in the right-hand member of the equation are of the order of $\varepsilon^{-\left(n_{\mathrm{e}}-1 / 2\right)}$ and turn out to be equal, so that the first-order asymptotic solution for the Eulerian perturbation of the gravitational potential is equal to zero. For larger values of $\tau_{\mathbf{s}}(r)$, the first term in the right-hand member of Eq. (18) is of the order of $\varepsilon$, while the second term tends to zero. Therefore, the first-order asymptotic solution becomes of the order of $\varepsilon$, in accordance with the order of the asymptotic solution $\Phi^{(c)}(r ; \varepsilon)$ which is uniformly valid from $r=0$ to a sufficiently large distance from $r=R$.

\section{The equation for the eigenfrequencies}

The equation for the eigenfrequencies results from the requirement that the first-order asymptotic solutions $\alpha^{(c)}(r ; \varepsilon)$ and $\alpha^{(\mathrm{s})}(r ; \varepsilon)$ must be continuous at any point at sufficiently large distances from the boundary points where they are both uniformly valid. At these distances, the Bessel functions $J_{\ell+1 / 2}(\tau)$ and $J_{n_{\mathrm{e}}+1}\left(\tau_{\mathrm{s}}\right)$ may be approximated by their asymptotic form for large values of their argument. The requirement is then that

$$
\begin{aligned}
& A_{0, c} \cos \left[\tau-(\ell+1) \frac{\pi}{2}\right] \\
& \quad=A_{0, \mathrm{~s}} \cos \left[\tau-\tau_{R}-\left(n_{\mathrm{e}}+\frac{3}{2}\right) \frac{\pi}{2}\right]
\end{aligned}
$$

and leads to the conditions

$$
\left.\begin{array}{c}
A_{0, c} \cos \left[(\ell+1) \frac{\pi}{2}\right] \\
-A_{0, \mathrm{~s}} \cos \left[-\tau_{R}+\left(n_{\mathrm{e}}+\frac{3}{2}\right) \frac{\pi}{2}\right]=0, \\
A_{0, c} \sin \left[(\ell+1) \frac{\pi}{2}\right] \\
+A_{0, \mathrm{~s}} \sin \left[-\tau_{R}+\left(n_{\mathrm{e}}+\frac{3}{2}\right) \frac{\pi}{2}\right]=0 .
\end{array}\right\}
$$

A necessary and sufficient condition for the system of equations to admit of solutions for the undetermined constants $A_{0, c}$ and $A_{0, \mathrm{~s}}$ that are different from zero is

$$
\begin{gathered}
\frac{[\ell(\ell+1)]^{1 / 2}}{\sigma} \int_{0}^{R} \frac{N\left(r^{\prime}\right)}{r^{\prime}} \mathrm{d} r^{\prime} \\
=\left(\ell+n_{\mathrm{e}}+2 \kappa+\frac{1}{2}\right) \frac{\pi}{2} .
\end{gathered}
$$

Here $\kappa$ takes the values $\kappa=1,2,3, \ldots$ and corresponds to the radial order of the $g^{+}$-mode considered. Equation (36) corresponds to the equation established earlier by Tassoul [1980, Eq. (123)] in the Cowling approximation. The reason for this agreement is that the equation for the eigenfrequencies results from the continuity of the divergence of the Lagrangian displacement and its first derivative and that, compared to the divergence of the Lagrangian displacement, the Eulerian perturbation of the gravitational potential is at least one order higher in the small expansion parameter $\varepsilon$ everywhere in the star. 


\section{Concluding remarks}

We conclude that the subseismic approximation results automatically from the first-order asymptotic theory for low-degree, low-frequency gravity modes in a spherically symmetric star developed by Smeyers et al. (1995). At the same order of asymptotic approximation, the Eulerian perturbation of the mass density is derived from the equation of mass conservation and is different from zero.

The subseismic approximation is a natural consequence of the first-order asymptotic theory in the main body of a star but not in a small region near the star's surface. The use of the subseismic approximation near a star's surface leads to an inadequate representation of lowfrequency gravity modes in that region, as was already observed by De Boeck et al. (1992).
Acknowledgements. The author gratefully acknowledges the comments of the referee, Dr. M. J. Clement, which have led to an improvement in the presentation of the paper.

\section{References}

De Boeck, I., Van Hoolst, T., \& Smeyers, P. 1992, A\&A, 259, 167

Dintrans, B., \& Rieutord, M. 2001, MNRAS, in press

Pekeris, C. L. 1938, ApJ, 88, 189

Smeyers, P., De Boeck, I., Van Hoolst, T., \& Decock, L. 1995, A\&A, 301, 105

Smylie, D., \& Rochester, M. G. 1981, Phys. Earth Plan. Int., 24,308

Smylie, D., Szeto, A., \& Rochester, M. G. 1984, Rep. Prog. Phys., 47, 855

Tassoul, M. 1990, ApJ, 358, 313 